\title{
Splenodiaphragmatic colonic interposition and left hemidiaphragmatic elevation in a patient undergoing robot-assisted radical prostatectomy: a case report
}

\begin{abstract}
Intestinal interposition occurs when a segment of the bowel is temporarily or permanently interposed between two organs. In most cases, this condition is asymptomatic and represents an incidental radiologic finding, but in rare cases abdominal and even respiratory symptoms have been described. We report the case of a 53-year-old man with normal BMI, candidate to robot-assisted radical prostatectomy (RARP) for high-risk prostate cancer, who had an incidental finding of splenodiaphragmatic colonic interposition and left hemidiaphragmatic elevation. These findings raised concern about possible problems for intraoperative ventilation, since the position of the patient during RARP and the use of pneumoperitoneum invariably restrict diaphragmatic and chest wall excursion, which can adversely affect respiratory gas exchange. Anyway, no problem occurred, and the procedure was carried out as usual with a $27^{\circ}$ Trendelenburg position and a slightly reduced pneumoperitoneum of $10 \mathrm{mmHg}$
\end{abstract}

Keywords: prostatectomy, colonic interposition, intestinal interposition, Chilaiditi
Volume 6 Issue 2 - 2018

\author{
Gabriele Cozzi,' Gennaro Musi,' Sarah \\ Alessi, ${ }^{2}$ Matteo Ferro,' Francesco Alessandro \\ Mistretta,' Laura Della Grazia, ${ }^{3}$ Marco \\ Venturino, ${ }^{3}$ Ottavio De Cobelli, ${ }^{1,4}$ \\ 'European Institute of Oncology, Division of Urology, Italy \\ ${ }^{2}$ European Institute of Oncology, Division of Radiology, Italy \\ ${ }^{3}$ European Institute of Oncology, Division of Anesthesiology, \\ Italy \\ ${ }^{4}$ Associate Professor of Urology, Università degli Studi di Milano, \\ Italy
}

Correspondence: Gabriele Cozzi, European Institute of Oncology, Division of Urology, Via Ripamonti, 435, 20I4I Milan, Italy, Tel +390257489367, Email gabriele.cozzi@ieo.it

Received: January 24, 2018 | Published: April 24, 2018
Abbreviations: RARP, robot-assisted radical prostatectomy; PSA, prostatic-specific antigen; mpMRI, multi-parametric magnetic resonance imaging; CXR, chest X-ray; FRC, functional reserve capacity

\section{Introduction}

Intestinal interposition occurs when a segment of the bowel is temporarily or permanently interposed between two organs. Hepatodiaphragmatic colonic interposition is termed Chilaiditi sign, while other conditions such as splenodiaphragmatic, splenorenal or gastropancreatic interposition are termed non-Chilaiditi sign. ${ }^{1}$ In most cases, this condition is asymptomatic and represents an incidental radiologic finding, but in rare cases abdominal and even respiratory symptoms have been described. ${ }^{2}$

The position of the patient during robot-assisted radical prostatectomy (RARP) invariably restricts diaphragmatic and chest wall excursion, which can adversely affect respiratory gas exchange, ${ }^{3}$ so the finding of a colonic interposition with hemidiaphragmatic elevation may rise some concern regarding intraoperative ventilation.

We present the case of a patient who had the incidental finding of a left colonic interposition with homolateral hemidiaphragmatic elevation and then underwent RARP.

\section{Case report}

A 53-year-old man with negative medical history and BMI of $26.14 \mathrm{~kg} / \mathrm{m}^{2}$ presented in January 2017 with apparently low-risk prostate cancer: his prostatic-specific antigen (PSA) was 10.8ng/ $\mathrm{ml}$ and a prostate biopsy demonstrated a Gleason score $3+3=6$ prostatic adenocarcinoma in 1 of 12 cores. Digital rectal examination was unremarkable (T1c). The patient was then offered an active surveillance program that he accepted. As part of the program, the patient underwent multi-parametric magnetic resonance imaging (mpMRI) of the prostate: the imaging showed a $16 \mathrm{~mm}$ anterior lesion that was highly suspicious for clinically significant disease. A MRIguided prostate biopsy was performed, and a Gleason score $4+4=8$ adenocarcinoma was detected. The patient was then addressed to surgical treatment and RARP was offered.

During standard preoperative workout, a chest X-ray (CXR) was performed, showing elevation of the left diaphragm due to splenodiaphragmatic colonic interposition (Figure 1). This finding raised concern about possible problems for intraoperative ventilation in steep Trendelenburg posture together with pneumoperitoneum. The situation was discussed with the patient, who was informed that a conversion to open surgery could have been possible in case of gas exchange problems due to the hemidiaphragmatic elevation.

At the time of surgery, pneumoperitoneum was inducted more slowly than usual, and kept at $10 \mathrm{mmHg}$. A $27^{\circ}$ Trendelenburg position was then gradually reached. Pneumoperitoneum was well tolerated and no problems related to ventilation occurred (Figure 2). RARP with bilateral extended pelvic lymph node dissection was performed as previously described; ${ }^{4}$ surgery lasted 2 hours and 40 minutes, estimated blood loss was $200 \mathrm{ml}$ and no intraoperative complications occurred. Post-operative course was unremarkable and the patient was discharged on $2^{\text {nd }}$ post-operative day. 


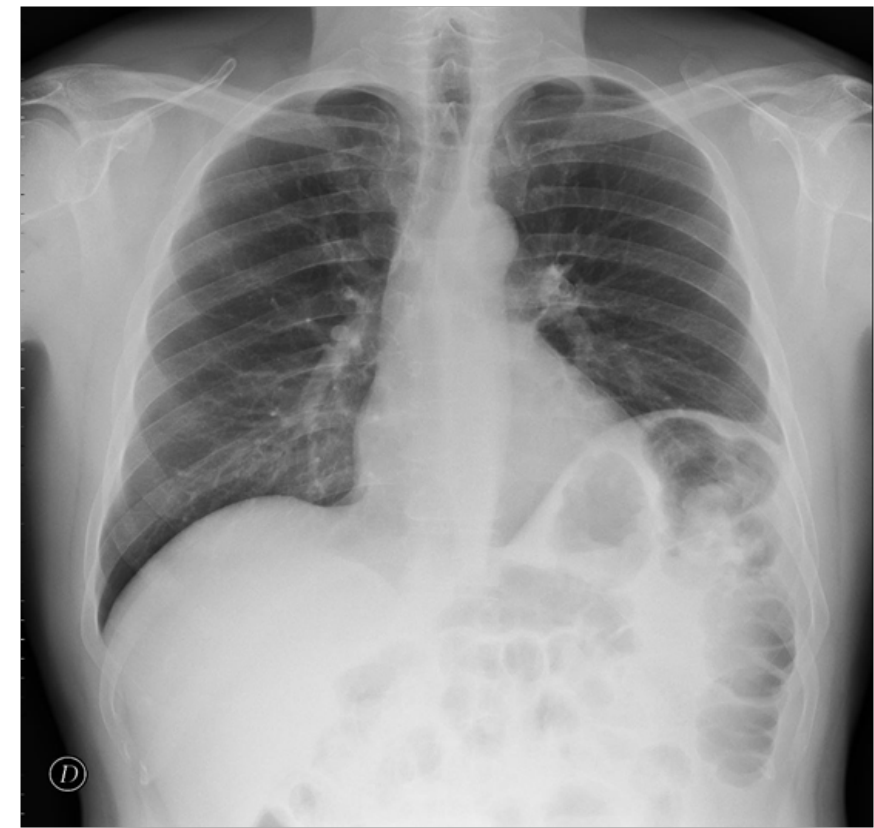

Figure I Chest X-ray showing elevation of the left hemidiaphragm due to splenodiaphragmatic colonic interposition.

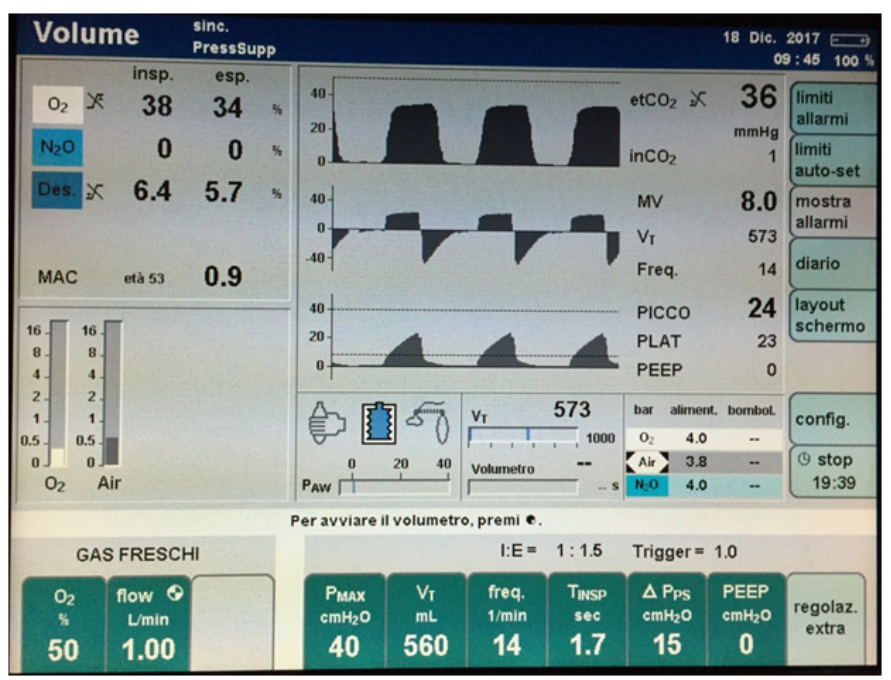

Figure 2 Anesthesia monitor showing normal peak pressure and end-Tidal $\mathrm{CO}_{2}$ values.

\section{Discussion}

To the best of our knowledge, this is the first reported case of a man with left colonic interposition and homolateral hemi-diaphragm elevation who underwent RARP.

Colonic interposition has an incidence of $0.25 \%$ to $0.28 \%$ worldwide with a marked male predominance (male to female, $4: 1){ }^{5}$ This condition is usually asymptomatic. ${ }^{2}$ When present, the most common symptoms are abdominal: recurrent pain in the epigastrium, nausea, vomiting, hiccups, constipation, flatulence, and a sensation of abdominal distension. Sometimes, respiratory symptoms, such as tachypnoea, shortness of breath, or chest pain, may occur. ${ }^{6}$

RARP has become the leading option for treating patients with clinically localized prostate cancer in the United States, and it has been progressively expanding in other countries. ${ }^{7}$

During RARP, the use of the steep Trendelenburg position causes abdominal contents to push the diaphragm cephalad along with all of the mediastinal structures. This cephalad movement reduces the lung's functional reserve capacity (FRC), decreases pulmonary compliance, and predisposes to atelectasis. ${ }^{8}$ Pneumoperitoneum is also known to impair respiratory mechanics: in fact, the European Association for Endoscopic Surgery recommends to avoid pressures higher than $12 \mathrm{mmHg}$ combined with Trendelenburg position because of decreased pulmonary compliance ${ }^{9}$ and a rise of more than $50 \%$ in peak and plateau pressures. ${ }^{8}$

These facts justified the concern about our patient, who presented with an elevated hemidiaphragm due to colonic interposition. In this case, despite these conditions, no problems related to gas exchange occurred; maybe a role has also been played by the normal BMI of the patient, since arterial oxygenation is significantly impaired in overweight patients under general anesthesia in Trendelenburg posture. ${ }^{10}$

According to our experience in this case, colonic interposition and hemidiaphragmatic elevation do not seem to pose a problem for patients with normal BMI undergoing robot-assisted pelvic surgery.

\section{Acknowledgements}

Gabriele Cozzi and Gennaro Musi equally contributed to the manuscript.

\section{Conflict of interest}

There are no financial or commercial interests to be acknowledged.

\section{References}

1. Weng WH, Liu DR, Feng CC, et al. Colonic interposition between the liver and left diaphragm - management of Chilaiditi syndrome: A case report and literature review. Oncol Lett. 2014;7(5):1657-60.

2. Fomin D, Baranauskaite V, Laima S, et al. Death Caused by Hepatodiaphragmatic Interposition of the Colon. $J$ Forensic Sci. 2017;62(1):247-9.

3. Lebowitz P, Yedlin A, Hakimi AA, et al. Respiratory gas exchange during robotic-assisted laparoscopic radical prostatectomy. J Clin Anesth. 2015;27(6):470-5.

4. Almeida GL, Musi G, Mazzoleni F, et al. Intraoperative frozen pathology during robot-assisted laparoscopic radical prostatectomy: can ALEXIS trocar make it easy and fast? J Endourol. 2013;27(10):1213-7.

5. Nair N, Takieddine Z, Tariq H. Colonic Interposition between the Liver and Diaphragm: "The Chilaiditi Sign". Can J Gastroenterol Hepatol. 2016;2016:2174704.

6. Fisher AA, Davis MW. An elderly man with chest pain, shortness of breath, and constipation. Postgrad Med J. 2003;79(929):180,3-4.

7. Montorsi F, Wilson TG, Rosen RC, et al. Best practices in robot-assisted radical prostatectomy: recommendations of the Pasadena Consensus Panel. Eur Urol. 2012;62(3):368-81. 
8. Gainsburg DM. Anesthetic concerns for robotic-assisted laparoscopic radical prostatectomy. Minerva Anestesiol. 2012;78(5):596-604.

9. Neudecker J, Sauerland S, Neugebauer E, et al. The European Association for Endoscopic Surgery clinical practice guideline on the pneumoperitoneum for laparoscopic surgery. Surg Endosc. 2002;16(7):1121-43.
10. Meininger D, Zwissler B, Byhahn C, et al. Impact of overweight and pneumoperitoneum on hemodynamics and oxygenation during prolonged laparoscopic surgery. World J Surg. 2006;30(4):520-6. 